\title{
Homonymous hemianopia: challenges and solutions
}

\section{Denise Goodwin}

Pacific University College of Optometry, Forest Grove, OR, USA

\author{
This article was published in the following Dove Press journal: \\ Clinical Ophthalmology \\ 22 September 2014 \\ Number of times this article has been viewed
}

\begin{abstract}
Stroke is the most common cause of homonymous hemianopia (HH) in adults, followed by trauma and tumors. Associated signs and symptoms, as well as visual field characteristics such as location and congruity, can help determine the location of the causative brain lesion. HH can have a significant effect on quality of life, including problems with driving, reading, or navigation. This can result in decreased independence, inability to enjoy leisure activities, and injuries. Understanding these restrictions, as well as the management options, can aid in making the best use of remaining vision. Treatment options include prismatic correction to expand the remaining visual field, compensatory training to improve visual search abilities, and vision restoration therapy to improve the vision itself. Spontaneous recovery can occur within the first months. However, because spontaneous recovery does not always occur, methods of reducing visual disability play an important role in the rehabilitation of patients with $\mathrm{HH}$.
\end{abstract}

Keywords: hemianopia, homonymous hemianopia, visual field defects, visual training, perimetry

\section{Introduction}

Homonymous hemianopia $(\mathrm{HH})$ involves vision loss on the same side of the visual field in both eyes. This type of visual field loss is indicative of a lesion involving the visual pathway posterior to the chiasm. HH can affect the ability to drive or read and may result in injuries due to falls or inability to navigate around obstacles. Identifying and managing these visual difficulties can have a significant effect on a patient's quality of life. This review covers the etiology, clinical examination findings, patient challenges, management options, and prognosis associated with $\mathrm{HH}$.

\section{Etiology}

The potential causes of $\mathrm{HH}$ are dependent on the age of the patient. The most common cause of $\mathrm{HH}$ in adults is stroke. Approximately $8 \%-10 \%$ of stroke patients have permanent $\mathrm{HH}$, and $52 \%-70 \%$ of hemianopias are caused by stroke. ${ }^{1,2}$ As the population ages and stroke patients live longer, the incidence of stroke and resultant $\mathrm{HH}$ is likely to increase. ${ }^{3}$

Other common causes of $\mathrm{HH}$ include traumatic brain injury (14\% of HH cases) and tumors (11\% of HH cases). ${ }^{1,4}$ Less common causes of $\mathrm{HH}$ are shown in Table $1 .{ }^{4-13}$

Temporary HH with spontaneous recovery may occur due to migraine, ${ }^{14}$ occipital, parietal, or temporal lobe seizures $;{ }^{15}$ or transient ischemic attack. ${ }^{16}$ Nonketotic hyperglycemia can cause $\mathrm{HH}$ that resolves with normalization of the blood sugar. ${ }^{6,17,18}$

The most common causes of $\mathrm{HH}$ in children aged $\leq 18$ years are tumors $(27 \%-39 \%)$, brain injury $(19 \%-34 \%)$, infarction $(11 \%-23 \%)$, and cerebral hemorrhage $(7 \%-11 \%){ }^{19,20}$
Correspondence: Denise Goodwin Pacific University College of Optometry, 2043 College Way, Forest Grove,

OR 97II6, USA

Tel +I (503) 3523070

Fax +I (503) 3522929

Email goodwin@pacificu.edu 
Table I Causes of homonymous hemianopia

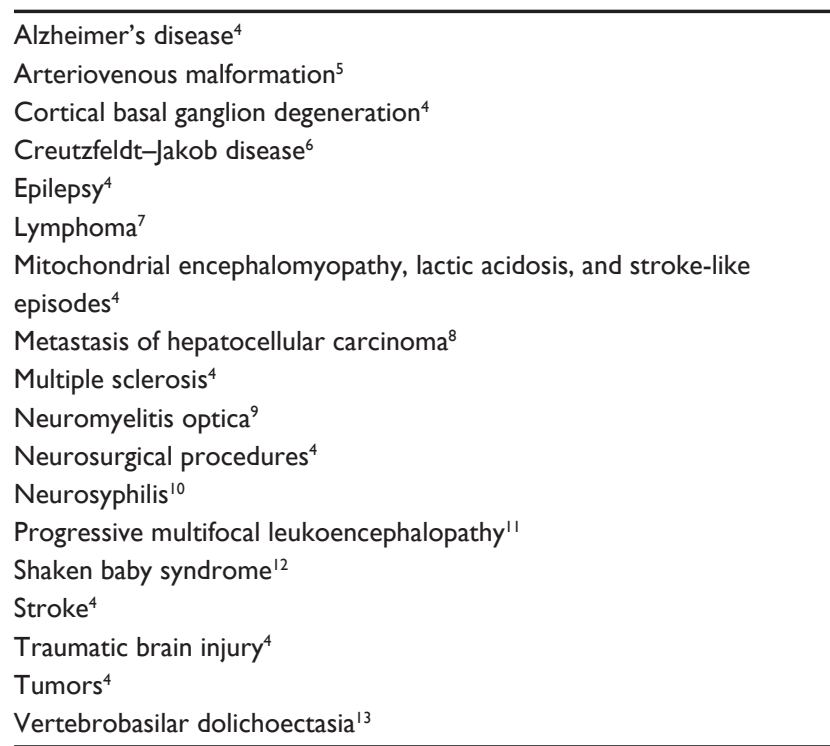

\section{Clinical evaluation}

Visual fields, particularly when correlated with other symptoms, provide valuable information regarding the location of brain lesions. Goldmann perimetry is useful in detecting neurologic visual field loss. Unfortunately, the equipment is not widely available, and testing requires a more skilled technician compared with automated perimetry. Humphrey automated perimetry is widely used for assessing visual field defects. The Swedish Interactive Threshold Algorithm (SITA) Fast is less sensitive than the SITA Standard method. SITA Fast testing can give reliable screening results, but because of the larger test-retest variability it may not be a good choice to monitor visual field loss over time. ${ }^{21}$ Also, quantitative comparison of different strategies should be avoided, as the mean deviation is higher with SITA Fast compared with SITA Standard. 22,23

Frequency doubling technology (FDT) perimetry can detect glaucomatous visual field damage earlier than standard perimetry by isolating specific types of ganglion cells. ${ }^{24}$ While these technologies are good at identifying patients with glaucoma, they have been shown to be inconsistent in finding neurologic visual field defects. Compared with Goldmann perimetry, the FDT C20 threshold protocol was found to detect hemianopic field loss less than half of the time. ${ }^{25} \mathrm{With}$ the use of a smaller stimulus size, the second-generation Matrix FDT has better correlation with standard perimetry when measuring neurologic disorders. ${ }^{26-28}$ However, the Matrix may still be less sensitive than standard automated perimetry in detecting $\mathrm{HH}$. Although not a statistically significant difference, hemianopic defects found with Goldmann perimetry were detected in $88 \%$ of cases with standard automated perimetry but only $69 \%$ of cases using the Matrix FDT. ${ }^{27}$

Confrontation visual field testing is not sensitive at detecting visual field loss, but it may be the only method available. Kerr et $\mathrm{al}^{29}$ evaluated 332 eyes prospectively to compare seven types of confrontation visual field tests. Finger counting was the least sensitive method, finding $0 \%$ of mild defects and $49 \%$ of severe defects. Overall, the most sensitive individual method of confrontation visual field testing is kinetic testing using a $5 \mathrm{~mm}$ red bead. This picks up 43\% of mild defects and $89 \%$ of severe defects. The overall sensitivity using the kinetic red bead is $74 \%$, but this improves to $78 \%$ when combined with static finger wiggle testing. Formal perimetry is necessary if there is a strong suspicion of visual field loss.

Unilateral damage to the retrochiasmal visual pathway results in bilateral vision loss affecting the contralateral visual field. The visual loss respects the vertical midline of the visual field. This should be differentiated from glaucomatous visual field loss, which does not respect the vertical midline but instead respects the horizontal midline. The most common location of lesions resulting in $\mathrm{HH}$ is the occipital lobe (45\%), followed by damage to the optic radiations (32\%). ${ }^{4}$ The remainder is caused by lesions of the optic tract $(10 \%)$, lateral geniculate nucleus (LGN) (1.3\%), or a combination of several areas (11\%).

A complete $\mathrm{HH}$ affects the entire hemifield of both eyes (Figure 1A). This can occur with a lesion anywhere posterior to chiasm and cannot be further localized based on visual field appearance alone. An incomplete $\mathrm{HH}$ spares at least part of the vision on the affected side and can be classified as either congruous or incongruous (Figure 1B and C). A congruous visual field defect is identical between the two eyes, whereas an incongruous defect differs in appearance between the eyes. For lesions behind the LGN, visual field defects are generally more congruous if the lesion is located more posteriorly along the visual pathway. However, exceptions do occur. Kedar et a ${ }^{30}$ demonstrated that although $84 \%$ of occipital lobe lesions produced congruous visual field defects, damage to the optic radiations was congruous in $59 \%$ of cases, and $50 \%$ of optic tract lesions produced congruous visual field loss. Despite this, the optic radiations remain the most common location for conditions resulting in incongruent visual field defects.

In addition to congruity, the shape and location of an incomplete HH can help localize the causative factor. Damage to the LGN will often result in one or more sectors of visual field loss, since the dorsal portion of the LGN contains fibers from the macular region, the lateral portion represents 


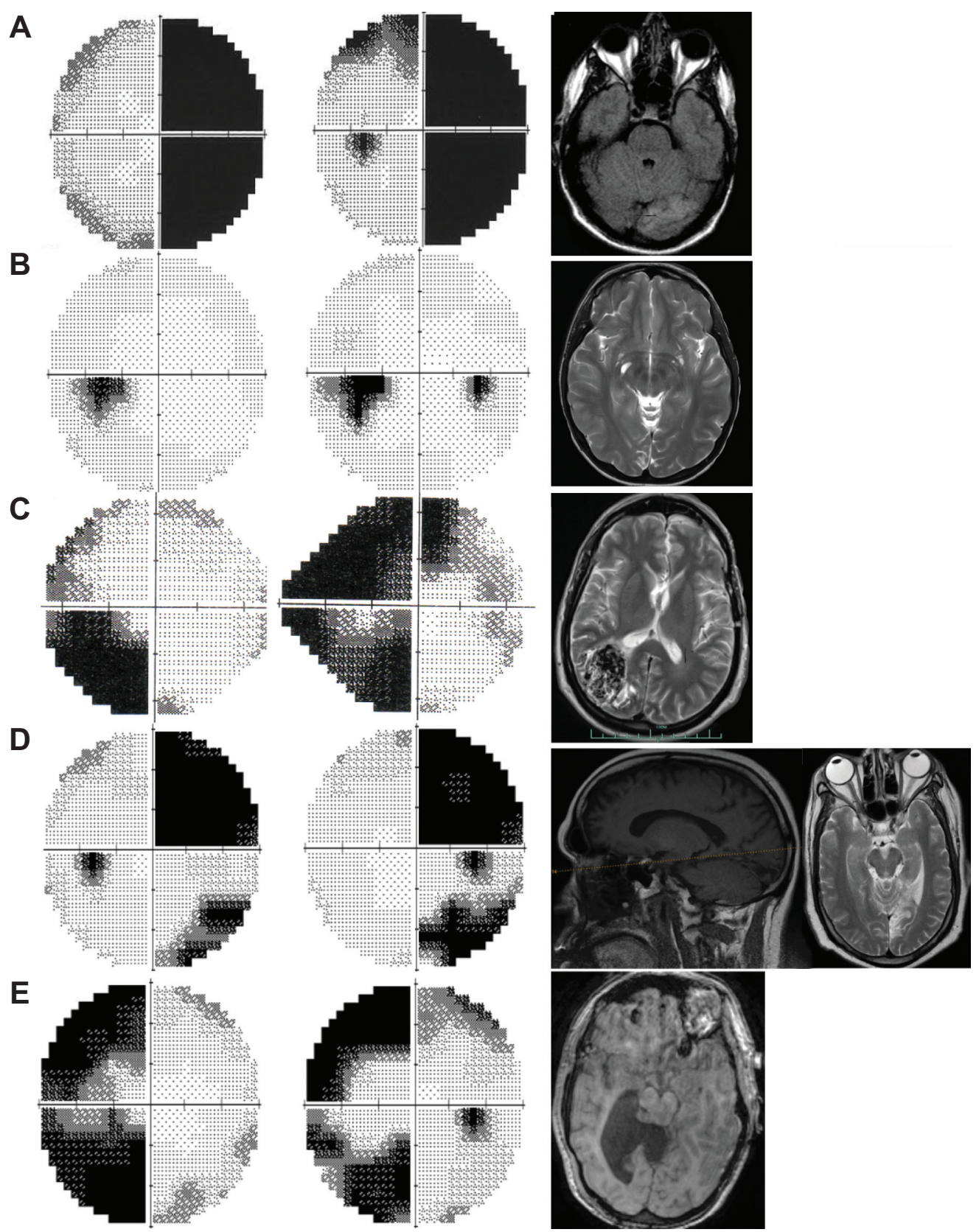

Figure I Examples of homonymous hemianopia with corresponding neuroimaging.

Notes: (A) Complete right homonymous hemianopia following a left occipital lobe stroke (axial TI magnetic resonance image [MRI] with contrast). (B) Left congruous homonymous hemianopia due to right occipital lobe encephalomalacia (axial T2 MRI). The patient also has a large Virchow-Robin space along the right optic tract, but it was felt the encephalomalacia was the more likely cause of visual field loss. (C) Left incongruous homonymous hemianopia due to right parietal lobe arteriovenous malformation (axial T2 MRI). (D) Right superior quadrantanopia following a stroke involving the left lingual gyrus and a right homonymous hemianopia involving the lower quadrant after a separate stoke involving the anterior portion of the left cuneus gyrus (sagittal TI MRI on left and axial T2 MRI on right; the dotted line on the left image indicates the level of the axial scan). (E) Left incongruous homonymous hemianopia with macular sparing due to hydrocephalus and subsequent shunt (axial TI MRI).

the superior visual field, and the medial portion represents the inferior visual field. Temporal lobe lesions tend to involve the superior visual field quadrant. In contrast, parietal lesions are more likely to cause inferior visual field defects with sloping borders superiorly. Lesions isolated to the upper, cuneus gyrus or the lower, lingual gyrus result in an inferior or superior quadrantanopia, respectively (Figure 1D).
Macular representation, found at the posterior pole of the occipital lobe, is disproportionately large. It is estimated that $50 \%-60 \%$ of the visual cortex represents $10^{\circ}-30^{\circ}$ of central vision. $^{31,32}$ Because of the large macular representation, as well as the dual blood supply to the posterior occipital lobe, sparing of the central $2^{\circ}-10^{\circ}$ of the visual field (Figure 1E) is commonly found with occipital lobe lesions. Macular 
sparring can also occur with lesions of the optic radiations or optic tracts. ${ }^{4}$ Even without macular sparing, an HH itself does not generally affect visual acuity. If visual acuity is reduced, an accompanying lesion involving the anterior visual pathway should be suspected.

Optic tract lesions can produce both complete and partial $\mathrm{HH}$. The presence of a relative afferent pupillary defect and band-shaped atrophy of the optic disc can help distinguish an optic tract lesion from damage located posterior to the LGN. When a complete $\mathrm{HH}$ is present, the relative afferent pupillary defect will be found in the eye with the temporal visual field loss (contralateral to the optic tract lesion). This may occur due to the increased number of decussating ganglion cell fibers compared with nondecussating fibers, or it may occur because the melanopsin-containing fibers found in the nasal retina have an increased sensitivity compared with those found in the temporal retina. ${ }^{33}$ Band-shaped atrophy, manifesting as pallor of the nasal and temporal optic nerve head (Figure 2), will also be seen in the eye contralateral to the optic tract lesion. Thinning of the retinal nerve fiber layer (NFL) that corresponds with the area of pallor can be seen with optical coherence tomography analysis of the NFL or ganglion cell complex. ${ }^{34}$ Here, retinal tissue associated with the temporal visual field loss, the NFL nasal to the disc, and the NFL temporal to the disc but nasal to the macula is reduced. Additionally, the superior and inferior NFL may be thinned in the eye ipsilateral to the optic tract lesion. Associated signs and symptoms may be present if the lesion

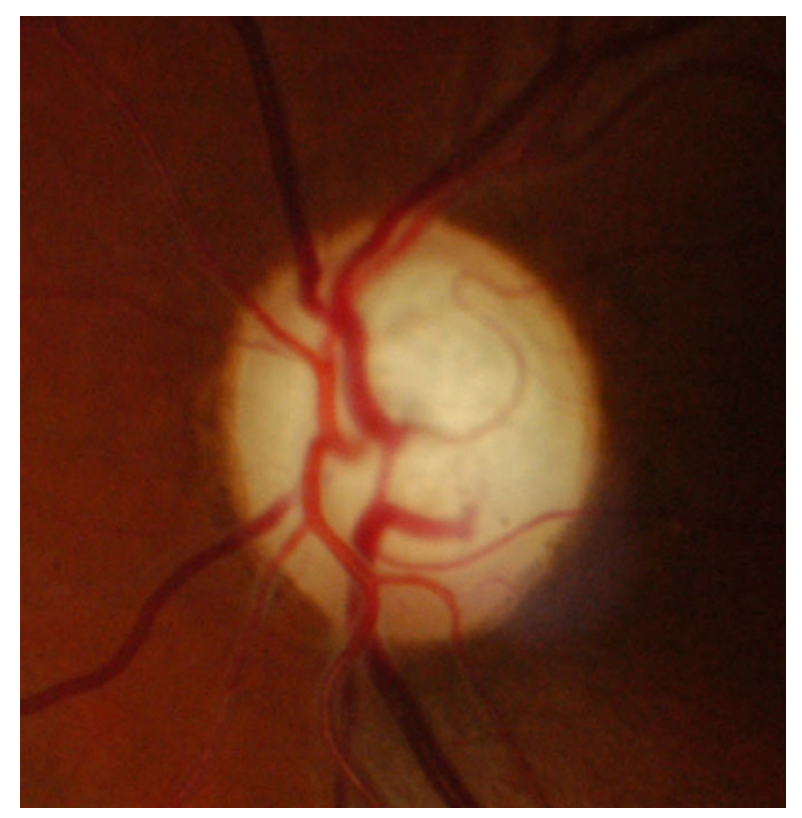

Figure 2 Band atrophy. Note the pallor of the nasal and temporal portions of the optic disc. affecting the optic tract extends to nearby structures. If the temporal lobe is involved, memory may be affected and seizures can occur. Damage to the adjacent cerebral peduncle can result in contralateral hemiparesis, and hypothalamic symptoms may be present.

Lesions affecting the optic radiations will not result in optic nerve pallor or pupillary defects. Either hemiparesis or hemianesthesia can result if the neighboring internal capsule is affected. Temporal lobe lesions are associated with memory and auditory problems, as well as seizures. Difficulty comprehending language (receptive aphasia) occurs if Wernicke's area is involved. Patients with parietal lobe lesions are often unaware of the visual field deficit. Sensory loss, agnosia, aphasia, apraxia, and difficulties with mathematics or writing may occur. Smooth pursuits may be impaired in the direction of the brain lesion, and patients often have difficulty maintaining fixation. Lesions involving the nondominant parietal lobe result in hemispatial neglect. Here, there is decreased awareness of stimuli contralateral to the side of the lesion.

$\mathrm{HH}$ is the main consequence of an occipital lobe lesion. Damage to the occipital lobe usually does not produce other neurologic manifestations. Some patients may experience photopsias or other hallucinations in the blind hemifield.

Reliable neurologic visual fields are easy to simulate with automated perimetry, even in those who have never before performed visual field testing. ${ }^{35} \mathrm{~A}$ spiral pattern with Goldmann perimetry, a tubular field with tangent screen testing, or a cloverleaf pattern with automated visual fields is indicative of functional visual loss. If nonorganic visual field loss is suspected, the visual field can be tested by asking the patient to saccade into the supposedly heminanopic field. The patient assumes you are testing eye movements, so they will not realize that they should not be accurately saccading to the target.

\section{Patient challenges}

Impairment of the visual field can be debilitating. In addition to the inability to drive, read, or navigate, the loss of independence and inability to enjoy leisure activities can have significant emotional and social implications. Understanding these restrictions can aid in rehabilitation and making the best use of remaining vision.

Inability to drive decreases independence, limits employment opportunities, and increases the risk of depression. ${ }^{36}$ In many areas of the US, patients with HH do not meet the legal driving requirements. A binocular visual field of at least $110^{\circ}$ is required in 27 states. ${ }^{37}$ Despite this, some continue 
to drive illegally, ${ }^{38,39}$ and 12 states have no minimum visual field requirement for driving. Therefore, it is important to be familiar with safety concerns as well as available options for these patients.

Drivers with $\mathrm{HH}$ have an impaired ability to detect and react to pedestrians on the side of the vision loss..$^{38,40,41}$ In a driving simulator, those with $\mathrm{HH}$ naturally make more head scans toward the blind side compared with normally sighted individuals; however, the scans tend to be of equal or smaller magnitude. ${ }^{40}$ Due, in part, to the smaller scans, simulated pedestrians are detected less than half of the time.

In an on-road driving evaluation, $41 \%$ of $\mathrm{HH}$ drivers had trouble controlling the vehicle position, $36 \%$ had problems adjusting their speed to traffic conditions, $27 \%$ did not respond adequately to unexpected events, and $27 \%$ had unusually bad driving maneuvers. ${ }^{5}$ Despite these problems, $73 \%-77 \%$ are deemed safe do drive in noninterstate conditions by driving rehabilitation specialists. ${ }^{5,42}$

Poor reading ability in those with $\mathrm{HH}$ may be due to reduced visual field, poor eye movements, or perceptual difficulties. Left-to-right readers with a right $\mathrm{HH}$ have particularly impaired reading abilities. For efficient reading, they must be able to see three to four letters to the left and seven to eleven letters to the right of fixation. ${ }^{43}$ These patients have trouble locating ensuing words and making systematic saccades to find those words. Additionally, eye movement patterns are disorganized, and there is prolonged fixation, reduced saccadic amplitude, and an increased number of regressive saccades. ${ }^{44}$ These combine to reduce reading speed and limit comprehension. Because parafoveal vision is used to plan saccades and obtain information about forthcoming words, those with $3^{\circ}-5^{\circ}$ of macular sparing tend to have minimal impairment of reading. ${ }^{45,46}$

Although not as severe, left-to-right readers with a left $\mathrm{HH}$ do have difficulty with reading. They have problems finding the subsequent line of text. ${ }^{44}$ Furthermore, since the first part of a word contains information to quickly identify the word, those with left-sided visual field defects have frequent reading errors. ${ }^{45,47}$

Hemianopia can cause difficulties evaluating the environment. This can result in disorientation, trouble crossing the street in traffic, bumping into objects, inability to detect hazards, and increased risk of falling. Patients with $\mathrm{HH}$ make more saccades toward the blind field, but the saccades are less accurate and systematic, resulting in much longer search times. ${ }^{48}$ This increased search time can explain the difficulties patients experience when attempting to find objects. Also, the slowed search patterns do not allow comprehension of the environment fast enough to avoid obstacles.

\section{Management options}

Management of patients with $\mathrm{HH}$ should involve a multidisciplinary approach. Vision training and low vision rehabilitation can improve specific visual deficiencies such as mobility or reading problems. Occupational therapy can help the patient navigate and function better in daily life. Psychological rehabilitation as well as social support can be critical in adjustment and improving quality of life. Other medical specialists may be involved in treating the underlying disorder.

Depending on the patient needs, treatment of visual deficits can include prismatic correction to expand the remaining visual field, compensatory training to improve visual search abilities, and vision restoration therapy to improve the vision itself. One type of therapy does not preclude other intervention methods. In fact, often, one therapy complements another. For example, compensatory training is often done in conjunction with prism therapy.

The goal of prism treatment is to expand the intact visual field. With the aid of prism, images that normally fall on the hemianopic retina are shifted so they become visible by the seeing portion of the retina. Although a number of methods of prescribing prism have been proposed, peripheral prism segments placed above and below the line of sight, originally described by Peli, ${ }^{49}$ have been the most successful in increasing the usable visual field while avoiding diplopia in primary gaze. Forty prism diopter segments placed unilaterally on the upper and lower parts of the spectacle lens provide up to $20^{\circ}$ of visual field expansion. ${ }^{50,51}$ The base is positioned toward the field loss on the lens that corresponds to the side of the hemianopia. This is often done with temporary press-on prisms. Alternatively, 57 prism diopters can be embedded permanently into the top and bottom portions of the glasses, providing $30^{\circ}$ visual field expansion.

These monocular prism segments can improve quality of life. ${ }^{2}$ A recent randomized controlled clinical trial demonstrated that treatment with these prisms improves mobility and avoidance of obstacles. ${ }^{50}$ Other studies have found that $29 \%-47 \%$ of patients continue to wear the glasses longterm. ${ }^{51,52}$

Obliquely oriented prism may have an advantage over horizontal prism because the oblique prism allows expansion of the central visual field. The image still falls on the peripheral retina so diplopia in primary gaze is avoided. Similar to the horizontal prism setup, two prism segments are placed 
with a $9 \mathrm{~mm}$ separation above and below the line of sight. The upper prism is placed on the lens that corresponds with the side of hemianopia with the base out and down at an angle of $30^{\circ}$. The lower prism is placed with the base out and up at an angle of $30^{\circ}$. Using 40 prism diopter oblique Fresnel prism segments, drivers with $\mathrm{HH}$ had improved response to unexpected hazards compared with those with sham prism. ${ }^{39}$ The increase in visual field obtained with 57 prism diopter obliquely placed prisms may allow patients with complete $\mathrm{HH}$ to drive legally in some states. Moss et $\mathrm{al}^{53}$ found an increase in the binocular field from $95^{\circ}$ to $115^{\circ}$ in one patient and from $82^{\circ}$ to $112^{\circ}$ in another patient. This would be sufficient to drive in over half of the states in the US.

The prisms are intended for distance viewing. However, if the patient has bifocal or progressive addition lenses, a small area can be cut out from the bottom of the Fresnel prism to allow reading. Otherwise, a separate pair of reading glasses is necessary.

Patients benefit from training regarding how to effectively use the prisms. Users should be instructed to look through the central portion of the glasses and scan their eyes as they normally would. They should not look through the prism, as this will result in diplopia. After detecting an object in the periphery, they should turn their head to fixate on the object.

Difficulties with the prisms include descending stairs, glare, inability to read with the prisms, and being startled when objects jump into the field of vision. ${ }^{52}$ Additionally, the optical quality of the press-on prisms deteriorates over time. Temporary prisms should be replaced every 3-4 months. Permanent prisms with better optic quality should be used if long-term wear is desired.

Compensatory training can improve eye and head scanning movements and help patients use their residual vision more efficiently to perform desired tasks. Training should include activities that improve general visual attention skills, increase the number and amplitude of saccades into the impaired hemifield, and develop a more organized pattern of eye movements. In addition, strategies to correct specific scanning deficits, such as improving reading or visual search abilities, should be employed.

One method of compensatory training starts training with the use of standardized colored lights along a horizontal plane. This system requires participants to use both head and eye movements. The exercises become more complex, resulting in patients being able to perform systematic and accurate search patterns. Mobility scanning strategies are then employed. These start in a structured environment and move to a complicated, dynamic environment. Some programs such as this teach a rigid systematic search strategy. Other programs employ searching for randomly placed targets among distractors. The patients are asked to fixate on the target as quickly as possible, moving only the eyes. No specific scanning strategy is suggested to the patient.

Teaching systematic scanning tactics results in more organized and efficient search times. ${ }^{48,54}$ The area in which patients can locate targets can be increased by up to $30^{\circ} .55,56$ More importantly, visual training can improve mobility and the ability to navigate while avoiding obstacles. ${ }^{57}$ In one study, $91 \%$ of participants were able to return to part-time work following compensatory training. ${ }^{56}$

Those who have a greater number and larger amplitude of head and eye movements have been shown to be safer drivers. ${ }^{58,59}$ In order to detect pedestrians, scans should be approximately $85^{\circ}$ toward the restricted visual field. Eye movement of $30^{\circ}$, in addition to head scanning of $55^{\circ}$, is necessary. ${ }^{40}$ The decreased scan amplitude often seen in those with $\mathrm{HH}$ may be a result of not having feedback from the peripheral vision to know how far to scan. Therefore, giving patients specific physical landmarks that will aid in knowing how far to turn their head may be helpful. ${ }^{40}$

Vision training can improve reading ability by decreasing errors and improving reading speed. ${ }^{44,60}$ Computer-based therapies that induce optokinetic nystagmus by having the patient read text scrolling from left to right have been shown to improve static reading speed by up to $46 \% .^{60,61}$ One example of this therapy can be downloaded for free here: http://www.readright.ucl.ac.uk.

Based on the concept of neuroplasticity, Vision Restorative Training (VRT) (NovaVision AG, Magdeburg, Germany) aims to regain visual function at the border of the visual field defect. This involves home-based training where suprathreshold light is presented to areas that border the visual field defect. Training is performed at least 1 hour each day for 6 months.

It is unclear whether VRT actually expands the usable visual field or whether unsteady fixation results in apparent visual field enlargement. When controlling for fixation, there was $<2^{\circ}$ of field expansion. ${ }^{62}$ An alternative explanation is that the visual field enlarges as the patient learns to improve attention or awareness of the vision loss. This theory is supported by research that found visual field improvement with compensatory visual field training alone. ${ }^{56,63}$ Regardless of the reason for field expansion, some patients do have improved reading speed following VRT. ${ }^{64}$ Unfortunately, the small visual field enlargement obtained 
is not likely to be sufficient for improved scanning of the environment..$^{55}$ The biggest downside to this treatment is the cost to the patient, which is around $\$ 6,000$ for the 6 months of treatment. ${ }^{65}$

Ideal rehabilitation is effective, simple to use, portable, and inexpensive. Given these criteria, optical compensation and compensatory training are viable options. Unfortunately, the cost and relatively small benefit of VRT make this option less practical. Most compensatory training can be performed at home using computer software. This has the advantage of being more cost-effective and provides access to more patients. Typically, therapy involves a mix of supervised office visits and home therapy. Unsupervised home training has been shown to be effective at improving reading performance, but not at improving avoidance of obstacles or hazard perception. ${ }^{66}$

\section{Prognosis}

Being familiar with the recovery pattern is important in patient education as well as in assessing the results of rehabilitation. Approximately $17 \%-19 \%$ of poststroke patients with total $\mathrm{HH}$ experience complete recovery within 1 month. ${ }^{67,68}$ In a different study, Zhang et al ${ }^{69}$ reported that $55 \%$ of HH patients have at least some improvement of the visual field within the first month. The prognosis was not significantly different for various causes of damage. Recovery decreases with increasing time from the injury, and most of the improvement occurs within the first 2 months ${ }^{69}$ Recovery is unlikely after 6 months unless the underlying cause resolves.

After 6 months, fixation patterns in adults become more dissimilar to those without $\mathrm{HH}$, indicating that with increased time from the onset of the $\mathrm{HH}$, patients may naturally compensate for the $\mathrm{HH} .{ }^{70}$ Patients with $\mathrm{HH}$ tend to concentrate on an area toward the side of the visual field defect rather than on the center of the image. ${ }^{71}$ They also make more saccades into the blind field compared with those with a normal visual field. ${ }^{70}$

Spontaneous recovery in children is reported to be similar to that found in adults. ${ }^{19}$ In addition, young children may adapt to the $\mathrm{HH}$ by developing an ipsilateral exotropia ${ }^{72}$ or a compensatory head turn toward the visual field defect. ${ }^{73}$ Exotropia with the eye deviating toward the side of the hemianopia can expand the visual field if anomalous correspondence is present. Unfortunately, adults do not develop this adaptation, but a patient with a congenital exotropia who develops an ipsilateral $\mathrm{HH}$ as an adult may benefit from an enlarged binocular visual field (Figure 3). In these cases, strabismus surgery should be avoided.

Charles Bonnet syndrome (CBS) involves recurrent, complex visual hallucination that occurs following vision loss. Individuals are generally aware that the images are not real, but they can cause significant anxiety. This has been reported in patients with HH. Generally, no treatment is warranted, but low-dose (5 mg daily) aripiprazole may help resolve the hallucinations and anxiety associated with CBS. ${ }^{74}$ Patients should be educated regarding the benign nature of CBS.

In some cases, motion perception remains despite damage to the occipital lobe (Riddoch phenomenon). These patients can localize and respond to stimuli despite the inability to consciously see the target. The underlying mechanism is not known, but projections directly between the extrastriate occipital cortex and the LGN or pulvinar nuclei may be responsible. ${ }^{75}$
A

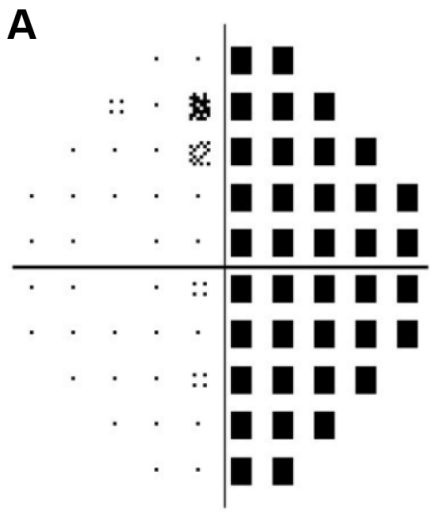

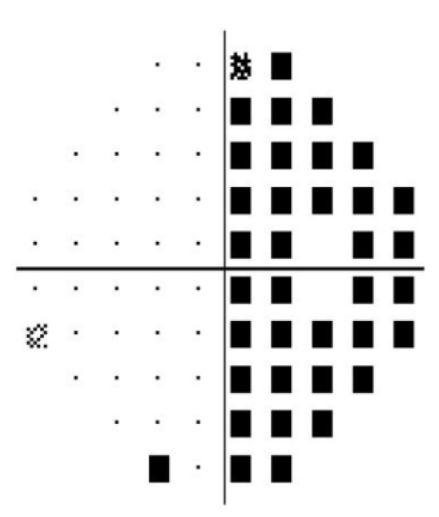

B

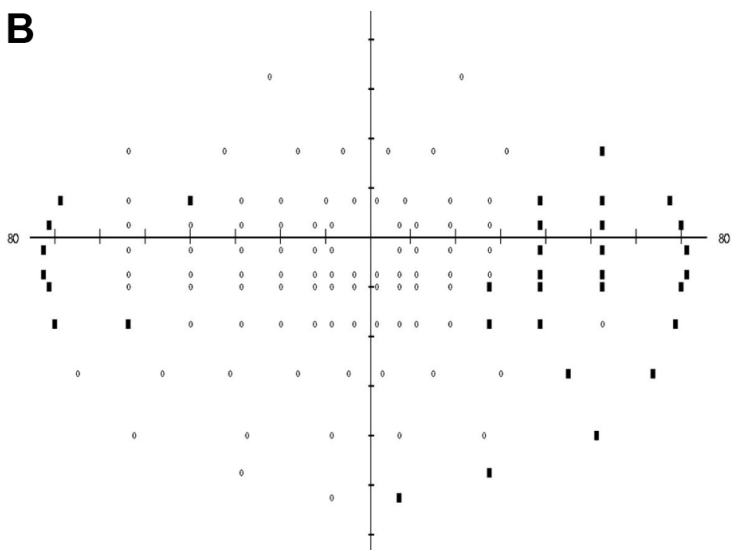

Figure 3 Example of how an ipsilateral exotropia can extend the usable visual field.

Notes: A 67-year-old male presented with complete right homonymous hemianopia following a stroke involving the occipital lobe. He had a long-standing 40 prism diopter right exotropia without amblyopia. Visual acuities were 20/20 in each eye. Monocular testing demonstrated a complete right homonymous hemianopia (A). With binocular visual field testing $(\mathbf{B})$, the patient was able to see an additional $30^{\circ}$ on the side of the hemianopia. 


\section{Conclusion}

$\mathrm{HH}$ can be disabling. Due to improving health care and increasing patient life span, the prevalence of $\mathrm{HH}$ will likely increase. Because spontaneous recovery does not always occur, methods of reducing visual disability play an important role in the rehabilitation of patients with $\mathrm{HH}$. Both optical and vision therapy can help to improve the ability to navigate safely within the environment and may enhance the capability of enjoying activities such as reading and driving.

\section{Disclosure}

The author reports no conflicts of interest in this work.

\section{References}

1. Zhang X, Kedar S, Lynn MJ, et al. Homonymous hemianopia in stroke. J Neuroophthalmol. 2006;26(3):180-183.

2. O'Neill EC, Connell PP, O'Connor JC, et al. Prism therapy and visual rehabilitation in homonymous visual field loss. Optom Vis Sci. 2011; 88(2):263-268.

3. Gilhotra JS, Mitchell P, Healey PR, et al. Homonymous visual field defects and stroke in an older population. Stroke. 2002;33(10):2417-2420.

4. Zhang X, Kedar S, Lynn MJ, et al. Homonymous hemianopias: clinical-anatomic correlations in 904 cases. Neurology. 2006;66(6): 906-910.

5. Elgin J, McGwin G, Wood JM, et al. Evaluation of on-road driving in people with hemianopia and quadrantanopia. Am J Occup Ther. 2010; 64(2):268-278.

6. Brazis PW, Lee AG, Graff-Radford N, et al. Homonymous visual field defects in patients without corresponding structural lesions on neuroimaging. J Neuroophthalmol. 2000;20(2):92-96.

7. Bruessow C, Karrer U, Gubler J, et al. Homonymous hemianopsia in a patient with Hodgkin's lymphoma in remission after BEACOPP chemotherapy. J Clin Oncol. 2012;30(12):e130-e132.

8. Hsu SY, Chang FL, Sheu MM, et al. Homonymous hemianopia caused by solitary skull metastasis of hepatocellular carcinoma. J Neuroophthalmol. 2008;28(1):51-54.

9. Romero RS, Gutierrez I, Wang E, et al. Homonymous hemimacular thinning: a unique presentation of optic tract injury in neuromyelitis optica. J Neuroophthalmol. 2012;32(2):150-153.

10. Iwamoto K, Aoyagi J, Kiyozuka T, et al. Neurosyphilis with unilateral optic tract lesion causing homonymous hemianopia. Neurologist. 2009; 15(6):345-346.

11. Herold TR, Jakl V, Graser A, et al. Hemianopia and visual loss due to progressive multifocal leukoencephalopathy in natalizumab-treated multiple sclerosis. Clin Ophthalmol. 2012;6:1131-1133.

12. Bevilacqua L, Kuczynski A, James-Galton M, et al. An inability to learn to read caused by shaken baby syndrome. BMJ Case Rep. 2014;2014.

13. Mortzos P, Sorensen TL. Visual loss, homonymous hemianopia, and unilateral optic neuropathy as the presenting symptoms of vertebrobasilar dolichoectasia. Case Rep Ophthalmol Med. 2013;2013:562397.

14. Goodwin D. Transient complete homonymous hemianopia associated with migraine. Optometry. 2011;82(5):298-305.

15. Ghosh P, Motamedi G, Osborne B, et al. Reversible blindness: simple partial seizures presenting as ictal and postictal hemianopsia. J Neuroophthalmol. 2010;30(3):272-275.

16. Lavallee PC, Cabrejo L, Labreuche J, et al. Spectrum of transient visual symptoms in a transient ischemic attack cohort. Stroke. 2013;44(12): 3312-3317.

17. Lavin $\mathrm{P}$, Donahue $\mathrm{S}$. Magnetic resonance imaging changes associated with transient homonymous hemianopia in patients with nonketotic hyperglycemia. Arch Ophthalmol. 2008;126(10):1467.
18. Taban M, Naugle RI, Lee MS. Transient homonymous hemianopia and positive visual phenomena in patients with nonketotic hyperglycemia. Arch Ophthalmol. 2007;125(6):845-847.

19. Kedar S, Zhang X, Lynn MJ, et al. Pediatric homonymous hemianopia. J AAPOS. 2006;10(3):249-252.

20. Liu GT, Galetta SL. Homonymous hemifield loss in childhood. Neurology. 1997;49(6):1748-1749.

21. Artes PH, Iwase A, Ohno Y, et al. Properties of perimetric threshold estimates from full threshold, SITA standard, and SITA fast strategies. Invest Ophthalmol Vis Sci. 2002;43(8):2654-2659.

22. Budenz DL, Rhee P, Feuer WJ, et al. Sensitivity and specificity of the swedish interactive threshold algorithm for glaucomatous visual field defects. Ophthalmology. 2002;109(6):1052-1058.

23. Nordmann JP, Brion F, Hamard P, et al. [Evaluation of the Humphrey perimetry programs SITA standard and SITA fast in normal probands and patients with glaucoma]. J Fr Ophtalmol. 1998;21(8):549-554. French.

24. Ferreras A, Polo V, Larrosa JM, et al. Can frequency-doubling technology and short-wavelength automated perimetries detect visual field defects before standard automated perimetry in patients with preperimetric glaucoma? J Glaucoma. 2007;16(4):372-383.

25. Wall M, Neahring RK, Woodward KR. Sensitivity and specificity of frequency doubling perimetry in neuro-ophthalmic disorders: a comparison with conventional automated perimetry. Invest Ophthalmol Vis Sci. 2002;43(4):1277-1283.

26. Yoon MK, Hwang TN, Day S, et al. Comparison of Humphrey matrix frequency doubling technology to standard automated perimetry in neuro-ophthalmic disease. Middle East Afr J Ophthalmol. 2012; 19(2):211-215.

27. Taravati P, Woodward KR, Keltner JL, et al. Sensitivity and specificity of the Humphrey matrix to detect homonymous hemianopias. Invest Ophthalmol Vis Sci. 2008;49(3):924-928.

28. Huang CQ, Carolan J, Redline D, et al. Humphrey matrix perimetry in optic nerve and chiasmal disorders: comparison with humphrey SITA standard 24-2. Invest Ophthalmol Vis Sci. 2008;49(3):917-923.

29. Kerr NM, Chew SS, Eady EK, et al. Diagnostic accuracy of confrontation visual field tests. Neurology. 2010;74(15):1184-1190.

30. Kedar S, Zhang X, Lynn MJ, et al. Congruency in homonymous hemianopia. Am J Ophthalmol. 2007;143(5):772-780.

31. McFadzean R, Brosnahan D, Hadley D, et al. Representation of the visual field in the occipital striate cortex. Br J Ophthalmol. 1994;78(3): 185-190.

32. Korogi Y, Takahashi M, Hirai T, et al. Representation of the visual field in the striate cortex: comparison of MR findings with visual field deficits in organic mercury poisoning (minamata disease). AJNR Am J Neuroradiol. 1997;18(6):1127-1130.

33. Kardon R, Kawasaki A, Miller NR. Origin of the relative afferent pupillary defect in optic tract lesions. Ophthalmology. 2006;113(8): 1345-1353.

34. Kanamori A, Nakamura M, Yamada Y, et al. Spectral-domain optical coherence tomography detects optic atrophy due to optic tract syndrome. Graefes Arch Clin Exp Ophthalmol. 2013;251(2):591-595.

35. Ghate D, Bodnarchuk B, Sanders S, et al. The ability of healthy volunteers to simulate a neurologic field defect on automated perimetry. Ophthalmology. 2014;121(3):759-762.

36. Ragland DR, Satariano WA, MacLeod KE. Driving cessation and increased depressive symptoms. J Gerontol A Biol Sci Med Sci. 2005; 60(3):399-403.

37. Foundation for Traffic Safety. Driver licensing policies and practices [webpage on the Internet]. Available at: http://lpp.seniordrivers.org/ lpp/index.cfm?selection=visionreqs. Accessed September 3, 2014.

38. Alberti CF, Peli E, Bowers AR. Driving with hemianopia: III. Detection of stationary and approaching pedestrians in a simulator. Invest Ophthalmol Vis Sci. 2014;55(1):368-374.

39. Bowers AR, Tant M, Peli E. A pilot evaluation of on-road detection performance by drivers with hemianopia using oblique peripheral prisms. Stroke Res Treat. 2012;2012:176806. 
40. Bowers AR, Ananyev E, Mandel AJ, et al. Driving with hemianopia: IV. Head scanning and detection at intersections in a simulator. Invest Ophthalmol Vis Sci. 2014;55(3):1540-1548.

41. Bowers AR, Mandel AJ, Goldstein RB, et al. Driving with hemianopia, I: Detection performance in a driving simulator. Invest Ophthalmol Vis Sci. 2009;50(11):5137-5147.

42. Wood JM, McGwin G Jr, Elgin J, et al. On-road driving performance by persons with hemianopia and quadrantanopia. Invest Ophthalmol Vis Sci. 2009;50(2):577-585.

43. Kerkhoff G. Neurovisual rehabilitation: recent developments and future directions. J Neurol Neurosurg Psychiatry. 2000;68(6):691-706.

44. Zihl J. Eye movement patterns in hemianopic dyslexia. Brain. 1995; 118(Pt 4):891-912.

45. Zihl J, von Cramon D. Visual field recovery from scotoma in patients with postgeniculate damage. A review of 55 cases. Brain. 1985; 108(Pt 2):335-365.

46. Papageorgiou E, Hardiess G, Schaeffel F, et al. Assessment of visionrelated quality of life in patients with homonymous visual field defects. Graefes Arch Clin Exp Ophthalmol. 2007;245(12):1749-1758.

47. Grunda T, Marsalek P, Sykorova P. Homonymous hemianopia and related visual defects: restoration of vision after a stroke. Acta Neurobiol Exp. 2013;73(2):237-249.

48. Zihl J. Visual scanning behavior in patients with homonymous hemianopia. Neuropsychologia. 1995;33(3):287-303.

49. Peli E. Field expansion for homonymous hemianopia by optically induced peripheral exotropia. Optom Vis Sci. 2000;77(9):453-464.

50. Bowers AR, Keeney K, Peli E. Randomized crossover clinical trial of real and sham peripheral prism glasses for hemianopia. JAMA Ophthalmol. 2014;132(2):214-222.

51. Bowers AR, Keeney K, Peli E. Community-based trial of a peripheral prism visual field expansion device for hemianopia. Arch Ophthalmol. 2008;126(5):657-664.

52. Giorgi RG, Woods RL, Peli E. Clinical and laboratory evaluation of peripheral prism glasses for hemianopia. Optom Vis Sci. 2009; 86(5):492-502.

53. Moss AM, Harrison AR, Lee MS. Patients with homonymous hemianopia become visually qualified to drive using novel monocular sector prisms. J Neuroophthalmol. 2014;34(1):53-56.

54. Mannan SK, Pambakian AL, Kennard C. Compensatory strategies following visual search training in patients with homonymous hemianopia: an eye movement study. J Neurol. 2010;257(11):1812-1821.

55. Bouwmeester L, Heutink J, Lucas C. The effect of visual training for patients with visual field defects due to brain damage: A systematic review. J Neurol Neurosurg Psychiatry. 2007;78(6):555-564.

56. Kerkhoff G, Munssinger U, Meier EK. Neurovisual rehabilitation in cerebral blindness. Arch Neurol. 1994;51(5):474-481.

57. Hayes A, Chen CS, Clarke G, et al. Functional improvements following the use of the NVT vision rehabilitation program for patients with hemianopia following stroke. Neuro Rehabilitation. 2012;31(1):19-30.

58. Wood JM, McGwin G Jr, Elgin J, et al. Hemianopic and quadrantanopic field loss, eye and head movements, and driving. Invest Ophthalmol Vis Sci. 2011;52(3):1220-1225.
59. Papageorgiou E, Hardiess G, Mallot HA, et al. Gaze patterns predicting successful collision avoidance in patients with homonymous visual field defects. Vision Res. 2012;6525-6537.

60. Spitzyna GA, Wise RJ, McDonald SA, et al. Optokinetic therapy improves text reading in patients with hemianopic alexia: a controlled trial. Neurology. 2007;68(22):1922-1930.

61. Ong YH, Brown MM, Robinson P, et al. Read-right: A "web app" that improves reading speeds in patients with hemianopia. J Neurol. 2012; 259(12):2611-2615

62. Kasten E, Bunzenthal U, Sabel BA. Visual field recovery after vision restoration therapy (VRT) is independent of eye movements: an eye tracker study. Behav Brain Res. 2006;175(1):18-26.

63. Lane AR, Smith DT, Ellison A, et al. Visual exploration training is no better than attention training for treating hemianopia. Brain. 2010; 133(Pt 6):1717-1728.

64. Gall C, Sabel BA. Reading performance after vision rehabilitation of subjects with homonymous visual field defects. PM R. 2012; 4(12):928-935.

65. Pelak VS, Dubin M, Whitney E. Homonymous hemianopia: a critical analysis of optical devices, compensatory training, and NovaVision. Curr Treat Options Neurol. 2007;9(1):41-47.

66. Aimola L, Lane AR, Smith DT, et al. Efficacy and feasibility of homebased training for individuals with homonymous visual field defects. Neurorehabil Neural Repair. 2014;28(3):207-218.

67. Ali M, Hazelton C, Lyden P, et al. Recovery from poststroke visual impairment: evidence from a clinical trials resource. Neurorehabil Neural Repair. 2013;27(2):133-141.

68. Gray CS, French JM, Bates D, et al. Recovery of visual fields in acute stroke: Homonymous hemianopia associated with adverse prognosis. Age Ageing. 1989;18(6):419-421.

69. Zhang X, Kedar S, Lynn MJ, et al. Natural history of homonymous hemianopia. Neurology. 2006;66(6):901-905

70. Pambakian AL, Wooding DS, Patel N, et al. Scanning the visual world: a study of patients with homonymous hemianopia. J Neurol Neurosurg Psychiatry. 2000;69(6):751-759.

71. Ishiai S, Furukawa T, Tsukagoshi H. Eye-fixation patterns in homonymous hemianopia and unilateral spatial neglect. Neuropsychologia. 1987;25(4):675-679.

72. Donahue SP, Haun AK. Exotropia and face turn in children with homonymous hemianopia. J Neuroophthalmol. 2007;27(4):304-307.

73. Paysse EA, Coats DK. Anomalous head posture with early-onset homonymous hemianopia. J AAPOS. 1997;1(4):209-213.

74. Chen CC, Liu HC. Low-dose aripiprazole resolved complex hallucinations in the left visual field after right occipital infarction (Charles Bonnet syndrome). Psychogeriatrics. 2011;11(2):116-118.

75. Jobke S, Kasten E, Sabel BA. Vision restoration through extrastriate stimulation in patients with visual field defects: a double-blind and randomized experimental study. Neurorehabil Neural Repair. 2009; 23(3):246-255.
Clinical Ophthalmology

\section{Publish your work in this journal}

Clinical Ophthalmology is an international, peer-reviewed journa covering all subspecialties within ophthalmology. Key topics include: Optometry; Visual science; Pharmacology and drug therapy in eye diseases; Basic Sciences; Primary and Secondary eye care; Patient Safety and Quality of Care Improvements. This journal is indexed on Submit your manuscript here: http://www.dovepress.com/clinical-ophthalmology-journa

\section{Dovepress}

PubMed Central and CAS, and is the official journal of The Society of Clinical Ophthalmology (SCO). The manuscript management system is completely online and includes a very quick and fair peer-review system, which is all easy to use. Visit http://www.dovepress.com/ testimonials.php to read real quotes from published authors. 